\title{
SYMMETRIES, VARIATIONAL PRINCIPLES, AND QUANTUM DYNAMICS
}

\author{
J. MANJAVIDZE AND A. SISSAKIAN
}

Received 15 October 2003

We describe the role of symmetries in formation of quantum dynamics. A quantum version of d'Alembert's principle is proposed to take into account the symmetry constrains more exact. It is argued that the time reversibility of quantum process, as the quantum analogy of d'Alembert's principle, makes the measure of the corresponding path integral $\delta$-like. The argument of this $\delta$-function is the sum of all classical forces of the problem under consideration plus the random force of quantum excitations. Such measure establishes the one-to-one correspondence with classical mechanics and, for this reason, allows a free choice of the useful dynamical variables. The analysis shows that choosing the action-angle variables, one may get to the free-from-divergences quantum field theory. Moreover, one can try to get an independence from necessity to extract the degrees of freedom constrained by the symmetry. These properties of new quantization scheme are vitally essential for such theories as the non-Abelian Yang-Mills gauge theory and quantum gravity.

\section{Introduction}

We learned with deep grief the news about the decease of Ilya Romanovich Prigogine. It was not only because he was one of the most outstanding scientists in the world, but also because many of us had close personal scientific ties with him and were influenced by his fascinating intellectual personality.

Nikolai Nikolaevich Bogoliubov and Ilya Romanovich Prigogine were both devoted to methods of statistical physics. Bogoliubov praised very highly the scientific contributions and the human character of Prigogine. The group of Prigogine closely cooperated with the group of Professor Ivanov from Joint Institute for Nuclear Research (JINR) and some other researchers.

One of us (Sissakian) is happy to say that he had several discussions with Prigogine about cooperation and scientific issues. He was always very amiable, was interested in modern Russia, but as for science, he was always strict. 
Although he was not feeling very well at the Solvay conference in Greece (2001), Prigogine listened with interest to our new research in statistical approach in the theory of multiple particle production and made a number of very important remarks which later on were of great use in our research.

Prigogine was an outstanding scientist, and contributed very much to both physics and chemistry. It is widely known that his methods are successfully applied even in other branches of science. He was profoundly confident that nature is a whole entity and its separation into physics, chemistry, and other branches is conventionality introduced by man.

The problem of "book-keeping" the quantum degrees of freedom till now looks like a primary task for modern theory of the arbitrary many degrees of freedom system. The point is that the non-Abelian gauge theory of Yang and Mills or Einstein gravity, both obey high symmetry, and the symmetry itself signifies an existence of a connection among various degrees of freedom, that is, the symmetry reduces the number of dynamical degrees of freedom. So, "book-keeping" means the procedure of counting the independent degrees of freedom.

Our aim is to discuss an attempt to formulate the perturbation theory for a nonequilibrium problem constrained by the symmetry. We note that the problem of defining the notion of equilibrium in dissipative systems is itself very important. For instance, interest in this problem is often aroused because the transition to equilibrium in the dissipative systems is understood as the tendency to establish a certain order [18].

This question has a fundamental background. It is known that the symmetry may improve solvability of the quantum problem. The hidden $O(4)$ symmetry solves the Coulomb problem [2,9] and, therefore, the semiclassical Bohr-Sommerfeld quantization rules are exact for this problem. Another example is the SL(2C) symmetry which solves the 2-dimensional sin-Gordon field theory so that the semiclassical approximation becomes exact $[4,5]$. More examples one can find in [7]. So, the symmetry can actually reduce the quantum degrees of freedom.

We should keep in mind this marvellous phenomenon although it is hard to hope that we always deal with such simple "completely integrable systems" [1], in which there are enough number of symmetry constrains to prevent quantum perturbations. However, we have to keep in mind such a possibility. What if, for example, the Einstein gravity is the purely classical theory because of its highest space-time symmetry. Sure, this assumption looks curious, though, it is most likely that Einstein was thinking it is so. But there is also another side of the "book-keeping" problem.

Note that general quantum field, due to the constrains, cannot be treated always as the dispersive medium, unlike the electromagnetic fields which are described as one, two, and more photon states. In this case the quantum electrodynamics fundamental method of spectral analysis cannot be applied. An example is the soliton quantization problem, where ordinary methods are not effective [10].

One needs other formalism, which takes into account the symmetry constrains.

\section{Variational principles}

The modern quantum theories are practically based on the classical Hamiltonian variational principle. It can be built into the quantum theories keeping in mind Bohr's 
"correspondence principle." Formally, this becomes possible thanks to an outstanding result of Dirac that the action $S(u)$ is a generator of displacements along the $u(t)$ trajectory. Then the transition amplitude of the initial state, $\mid$ in $\rangle$, into the final one, $\mid$ out $\rangle$, is an exponent: $\langle$ in $|$ out $\rangle \sim \exp \{i S(u)\}$. The wave nature of quantum dynamics is realized then as a sum over all trajectories $u(t)$.

The simplest way of definition of this sum is based on the Ferma's principle and uses the basis of geometrical optics. A generalization represents an equivalent to the Hamiltonian variational principle. In this frame the dynamics is realized on the actions extremum, that is, it is supposed that the physical trajectory $u_{c}(t)$ is a solution of

$$
\frac{\delta S(u)}{\delta u(t)}=0
$$

This approach of "book-keeping" deviations from $u_{c}$ is known as the WKB method [19].

A weak point of this variational principle, as it is known from classical mechanics, is that it demands in a cumbersome way that the (symmetry) constrains be introduced into the formalism [8]. The formal problem is to extract the dynamical, that is, independent, degrees of freedom. In the quantum case this leads to implementation of the FaddeevPopov ansatz [8] which separates the dynamical degrees of freedom from symmetrical ones. Unfortunately, this method is unacceptable for non-Abelian gauge theory (in case the corresponding Yang-Mills fields are sufficiently strong [11], e.g., if the soliton-like excitation is considered) and for gravitational field [6].

\section{D'Alembert's variational principle}

We propose to use d'Alembert's variational principle which easily "absorbs" the constrains, as is known from the classics. For this reason, it is of a definite value for us.

Indeed, d'Alembert's variational principle means that the virtual deviation $\varepsilon(t)$ does not produce any work, $F(u) \varepsilon$, since the mechanical motion is time reversible. So, for mechanical systems $F(u) \mathcal{\varepsilon}(t)=0$, and, as soon as $\varepsilon(t)$ is an arbitrary function, one gets a condition that the sum of all the forces should be equal zero, $F(u)=0$. This equality may be treated as the equation of motion for $u(t)$ since $F(u)$ includes also the "force of inertia," $\sim \ddot{u}(t)$. If there are constrains in the system considered, then they have to be included along with other forces into $F(u)[1]$.

It should be noted here that, as follows from d'Alembert's principle, the solution of equation of motion $u_{c}$ already "absorbs" all the constrains. Therefore, it is enough to count all possible forms of $u_{c}$ which are fixed by the integration constants.

Therefore, the mechanics built on d'Alembert's variational principle seems to be most useful for us. The connection among various degrees of freedom, as well as constrains, is naturally included into the prescription that the mechanical motion must be time reversible.

The time reversibility may be achieved in quantum theories, for instance, considering loop trajectory with the transition amplitude $R=\langle$ in $|$ in $\rangle$. In the meantime, to define the integral over particle momentum unambiguously, one needs to implement the $i \epsilon$ prescription of Feynman. 
In particle physics the $i \epsilon$-prescription has been chosen so that a wave must disappear in remote future. Note that only such definition conserves a total probability. But it is evident that such a wave process is not time reversible. Therefore, even the simple loop trajectory cannot be considered as an example of time-reversible wave motion: due to the $i \epsilon$-prescription, the loop amplitude is complex, that is, it is not a singlet of complex conjugation operator.

This example creates an impression that quantum mechanics is time irreversible and, therefore, the d'Alembert's variational principle cannot be claimed for it. But then one may ask where is the quantum correspondence principle?

The answer is in fact that the amplitude $R$ is not a measurable quantity while only the product

$$
\left.\rho(\text { in }, \text { out })=\langle\text { in }| \text { out }\rangle\langle\text { out }| \text { in }\rangle=\langle\text { in }| \text { out }\rangle\langle\text { in }| \text { out }\rangle^{*}=\mid\langle\text { in }| \text { out }\right\rangle\left.\right|^{2}
$$

is measured experimentally, that is, it only has to be time-reversible quantity [12]. Note here that to define the amplitude $\langle$ in $|$ out $\rangle$, the $(+i \epsilon)$-prescription must be used, as well as for $\langle$ out $|$ in $\rangle=\langle$ in $|$ out $\rangle^{*}$, the $(-i \epsilon)$-prescription is applied. Therefore, just $\rho$ (in, out) describes the time-reversible process and this removes the contradiction with correspondence principle. We now consider what this gives us.

\section{Dirac functional measure}

Following Dirac, one writes

$$
\rho \sim e^{i S\left(u_{+}\right)} e^{-i S\left(u_{-}\right)} .
$$

Note that $u_{+}$and $u_{-}$are two completely independent trajectories and, by definition, the total action $\left\{S\left(u_{+}\right)-S\left(u_{-}\right)\right\}$is defined so that it describes closed-path motion.

To introduce d'Alembert's variational principle, one needs to distinguish between the physical trajectory $u(t)$ and the virtual deviation $\varepsilon(t)$ from it. Therefore, it is naturally that

$$
u_{ \pm}(t)=u(t) \pm \varepsilon(t)
$$

and the integrations over $u(t)$ and $\varepsilon(t)$ are taken independently [12]. Noticing that (i) the closed-path motion is already described and (ii) the end-points (in, out) cannot vary, the integration over $\varepsilon(t)$ must be performed with boundary conditions for initial $t_{i}$ and final $t_{f}$ times: $\varepsilon\left(t_{i}\right)=\varepsilon\left(t_{f}\right)=0$. Then, in the expanding in $\varepsilon(t)$,

$$
S(u+\varepsilon)-S(u-\varepsilon)=\left.\{S(u+\varepsilon)-S(u-\varepsilon)\}\right|_{\varepsilon=0}+2 \operatorname{Re} \int_{t_{i}}^{t_{f}} d t \frac{\delta S(u)}{\delta u(t)} \varepsilon(t)+U(c, \varepsilon),
$$

one may omit for the moment higher $\varepsilon(t)$-terms noted here by $U(u, \varepsilon)$. The first term is important if the classical trajectory is a periodic function [12]. The resulted integral over $\mathcal{E}(t)$ gives the functional $\delta$-function

$$
\int \prod_{t} d \varepsilon(t) \exp \left\{2 i \operatorname{Re} \int_{t_{i}}^{t_{f}} d t \frac{\delta S(u)}{\delta u(t)} \varepsilon(t)\right\}=\prod_{t}^{\prime} \delta\left(\frac{\delta S(u)}{\delta u(t)}\right) .
$$


This equality means that the continuum of contributions with $\delta S(u) / \delta u(t) \neq 0$ are cancelled in the integral independently of the shape of the function $u(t)$. It is the result of destructive interference among divergent $e^{+i S\left(u_{+}\right)}$and convergent $e^{-i S\left(u_{-}\right)}$waves. Note that this complete cancellation is actually a result of the time reversibility.

The dynamical equilibrium, when $\delta S(u) / \delta u(t)=0$, is the only surviving one, and in this case the integral (4.4) is infinite. This is expressed by $\delta$-function in (4.4). Therefore, integration over virtual deviation, $\varepsilon(t)$, leads to the Dirac $\delta$-like measure and the later defines the complete set of contributions in the integrals over the trajectory $u(t)$ [12].

Returning to d'Alembert's variational principle, one can notice that, formally, the product $\Upsilon=\varepsilon \delta S(u) / \delta u$ in the exponent in (4.4) represents "virtual work." But in the considered wave process case $\Upsilon \neq 0$, that is, the quantum virtual deviations can produce some work. However, as follows from (4.4), the "physical" trajectory is defined by the classical Lagrange equation (2.1) and, therefore, it is the trajectory where dynamical equilibrium is achieved.

Thus, we get a quantum version of d'Alembert's variational principle. It relies on the general principle of quantum theories that only the time-reversible amplitude module is a measurable quantity.

Furthermore, this formalism allows including external forces $[13,17]$. Thus, if $U(u, \varepsilon)$ takes into account the higher nonlinear in $\varepsilon(t)$ terms, then the strict path integral representation of $\rho$ is given by

$$
\rho=e^{-i K(j \varepsilon)} \int \prod_{t}^{\prime} d u(t) \delta\left(\frac{\delta S(u)}{\delta u(t)}+j(t)\right) e^{i U(u, \varepsilon)}
$$

where the operator

$$
2 \mathbf{K}(j \varepsilon)=\operatorname{Re} \int d t \frac{\delta}{\delta j(t)} \frac{\delta}{\delta \varepsilon(t)}
$$

generates a quantum perturbation series. The interaction functional $U(u, \varepsilon)$ can be easily discovered from the Lagrangian. At the very end, one should take in (4.5) the auxiliary variables $j$ and $\varepsilon$ to be equal to zero.

So, one can generate the quantum perturbations including the random force $j(t)$ being external to the classical system. This is an authentic indication that our formulation of quantum wave mechanics resembles the classical mechanics built on d'Alembert's variational principle base.

\section{Transition to the collective variables}

A generalization of the representation (4.5) to the field-theory case is straightforward. One should simply replace $u(t)$ by $u(x, t)$, where $x$ is the space coordinate and $t$ is the time $[15,16]$. The formalism does not depend on the type of field considered.

It must be noticed first of all that the functional $\delta$-function in (4.5) establishes one-toone correspondence among quantum and classical descriptions [12]: the quantum case introduces the random force $j(x)$ into the system influence via the Gaussian operator 
$\mathbf{K}(j \varepsilon)$. Therefore, if we know the general solution $u_{c} \neq 0$, then all quantum states can be counted, varying the integration constants as the dynamical variables.

This correspondence is extended so that it allows involving such a powerful method of classical mechanics as the method of transformation of dynamical variables. It should be underlined that this method cannot be adopted on the time-irreversible quantum amplitudes level $[13,16,17]$.

The most useful variables are the (action, angle)-type ones [14, 15] (in definite sense, this type of variables plays the same role as the "collective coordinates" of Bogoliubov [3]). In these terms the world line of classical system (with enough constrains) moves over (Arnold's) hypertorus with radii being equal to the action variable and its location is defined by the angle variables. In the quantum case, the action of the operator $\exp \{-i \mathbf{K}\}$ leads to the Gaussian thrilling of the hypertorus. This vibration may be described in terms of random fluctuation of the action and angle variables and so that quantum perturbation gets up not in the functional space of field $u(x, t)$.

A transformation to the special-type variables, namely, to a set of the integration constants, has been proposed in $[13,14,15,16,17]$. Note that if there is not enough constrains, then the system leaves the hypertorus surface and any mapping becomes meaningless. Such a situation is realized, for example, in quantum electrodynamics, which is gauge invariant, but this is not enough for the hypertorus formation. In this case the ordinary method of the Faddeev-Popov ansatz is effective.

It should be stressed that the discussed formulation of the theory is necessary and sufficient as well. The necessity follows from the fact that, in the situation of general position, the dynamics should be realized on Arnold's hypertorus [12]. The sufficiency follows from the fact that the fluctuations are defined on the Gaussian measure and so they cover the (action, angle) phase space completely.

We call such perturbation theory the topological theory. Indeed, the topological properties are crucial since if a trajectory on the hypertorus has definite winding number (topological charge), then the probability that after random quantum walking, the system returns to the same (or shifts on a constant winding number) position is equal to zero. Therefore, such systems have to be exactly semiclassical. The quantitative prove of this conclusion is given in $[13,16,17]$.

In the case if there is no topological charge, the system "freely" moves along the hypertorus and can be quantum. It is evident that the Yang-Mills in the real-time metric [14] and the gravitation are just such kind of theories.

The above-described transformation to the (action, angle)-type variables reduces the field-theoretical problem to the quantum mechanical one. Generally, such a mapping is singular but it is possible to isolate these infinities (they look like divergent integrals) and remove them via the normalization. This observation is extremely important since as a result we get the quantum mechanics theory which is free from divergences. One can mention that isolating the divergences and then cancelling them, the renormalization was already performed. This property is intriguing since the finiteness of the theory was attained without introduction of new fields, for example, supersymmetry partners.

It is understandable that there is no necessity to distinguish between the symmetry and dynamical degrees of freedom in new formulation of theory. Indeed, the quantum 
fluctuations are realized in the (action, angle) factor space and this does not touch the action symmetry. As a result, the perturbation series consists of transparently invariant terms [14].

\section{Conclusions}

We would like to note the following fact being important from the cognition point of view. It is remarkable that restricting the problem and computing the module of amplitude, that is, leaving the phase of amplitude undefined, the remarkable progress is shown to be achieved. So, we can say that, for instance, the Yang-Mills theory exists (see http://www.claymath.org/Millennium_Prize_Problems/ concerning more details on the question of existence of the Yang-Mills theory) as the theory of observables since in this restricted frame (i) it is free from the divergences and (ii) there is no problems [14] of necessity to distinguish between the dynamical and the symmetry degrees of freedom.

However, finally, it must be admitted that one does not know whether the time-reversibility principle, that is, the quantum d'Alembert's principle, can be applied to the Einstein gravity. If yes, then no gravitational waves are expected to be observed despite the fact that the gravity would be the quantum theory and, moreover, such theory could be free from divergences. The point is that, most likely, the gravity does not represent the dispersive media. This follows from the fact that the equation for metrics has a nontrivial solution.

\section{Acknowledgments}

The authors thank Kadyshevskii for constant interest in the above approach. The results have been discussed at different times with Baldin, Dremin, Kuvshinov, Matveev, Prigogine, to whom the authors are happy to express their gratitude.

\section{References}

[1] V. I. Arnold, Mathematical Methods of Classical Mechanics, Graduate Texts in Mathematics, vol. 60, Springer-Verlag, New York, 1978.

[2] V. Bargman, Zur theoria des wasserstoffatoms, Z. Phys. 99 (1976), 576-582 (German).

[3] N. Bogoliubov and S. Tyablikov, Approximate method of finding of lowest energy levels in metal, Sov. ZhETP 19 (1949), 256-268.

[4] R. Dashen, B. Hasslacher, and A. Neveu, Nonperturbative methods and extended-hadron models in field theory. I. Semiclassical functional methods, Phys. Rev. D 10 (1974), 4114-4129.

[5] _ Nonperturbative methods and extended-hadron models in field theory. II. Twodimensional models and extended hadrons, Phys. Rev. D 10 (1974), 4130-4138.

[6] B. DeWitt and C. Molina-París, Quantum gravity without ghosts, Modern Phys. Lett. A 13 (1998), no. 30, 2475-2479.

[7] J. S. Dowker, Quantum mechanics on group space and Huygens' principle, Ann. Physics 62 (1971), 361-382.

[8] L. D. Faddeev and V. N. Popov, Feynman diagrams for the Yang-Mills field, Phys. Lett. B 25 (1967), 29-30.

[9] V. Fock, Zur Theorie des Wasserstoffatoms, Z. Phys. 98 (1935), 145-154 (German).

[10] R. Jackiw, C. Nohl, and C. Rebbi, Classical and semiclassical solutions of the Yang-Mills theory, Particles and Fields (Proc. Banff Summer Inst., Banff, Alta., 1977) (D. H. Boal and A. N. Kamal, eds.), Plenum, New York, 1978, pp. 199-258. 
212 Symmetries, variational principles, and quantum dynamics

[11] G. W. Mackey, Induced Representations of Groups and Quantum Mechanics, W. A. Benjamin, New York, 1968.

[12] J. Manjavidze, On the connection between classical and quantum descriptions, Soviet J. Nuclear Phys. 45 (1987), no. 3, 442-446.

[13] Topology and perturbation theory, J. Math. Phys. 41 (2000), no. 8, 5710-5734.

[14] J. Manjavidze and A. Sissakian, Quantization of solitons in coset space, J. Math. Phys. 42 (2001), no. 1, 641-658.

[15] — Very high multiplicity hadron processes, Phys. Rep. 346 (2001), no. 1, 1-88.

[16] _ Yang-Mills field quantization in the factor space, J. Math. Phys. 42 (2001), no. 9, 41584180.

[17] A field theory description of constrained energy-dissipation processes, Theoret. and Math. Phys. 130 (2002), no. 2, 153-197.

[18] G. Nicolis and I. Prigogine, Self-Organization in Nonequilibrium Systems: From Dissipative Structures to Order Through Fluctuations, John Wiley \& Sons, New York, 1977.

[19] R. Rajaraman, Solitons and Instantons, North-Holland Publishing, Amsterdam, 1982.

J. Manjavidze: Joint Institute for Nuclear Research (JINR), 141980 Dubna, Moscow Region, Russia Current address: Institute of Physics, 380077 Tbilisi, Georgia

E-mail address: joseph@nusun.jinr.ru

A. Sissakian: Joint Institute for Nuclear Research (JINR), 141980 Dubna, Moscow Region, Russia E-mail address: sisakian@jinr.ru 


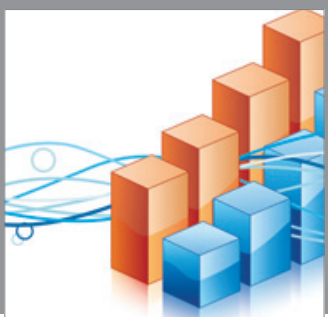

Advances in

Operations Research

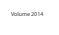

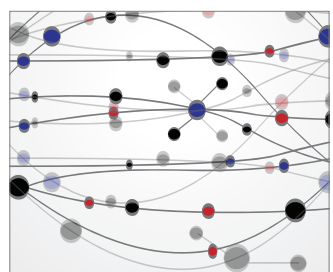

\section{The Scientific} World Journal
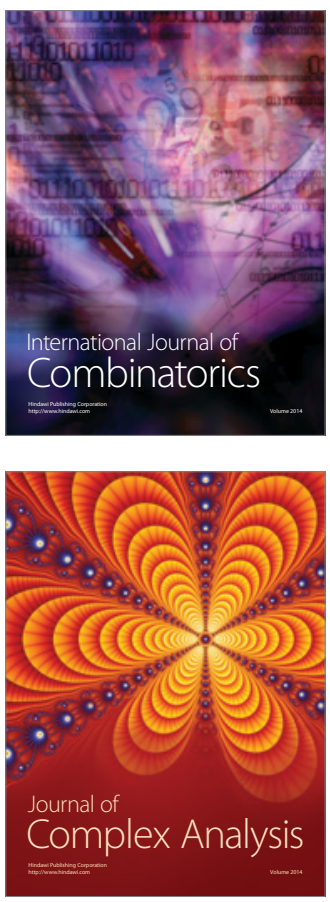

International Journal of

Mathematics and

Mathematical

Sciences
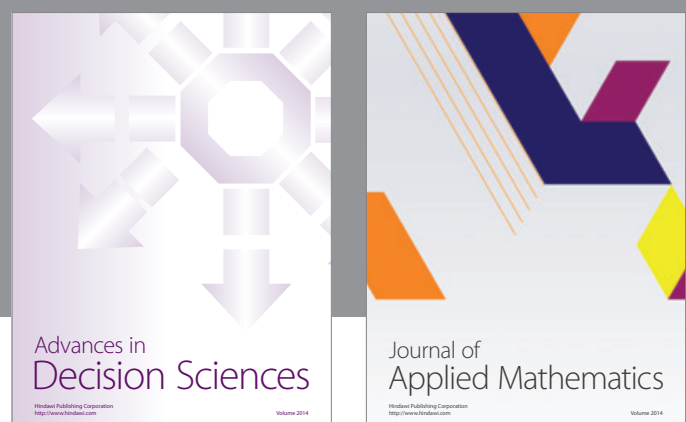

Journal of

Applied Mathematics
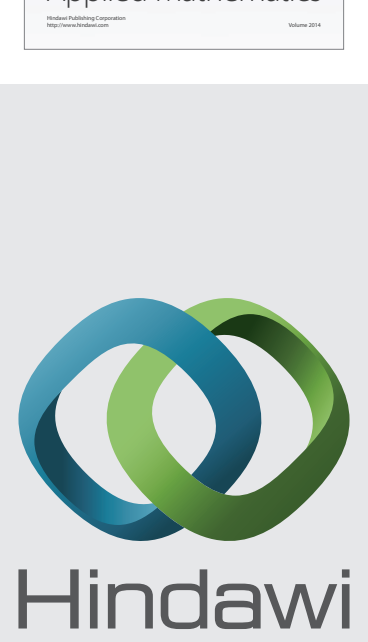

Submit your manuscripts at http://www.hindawi.com
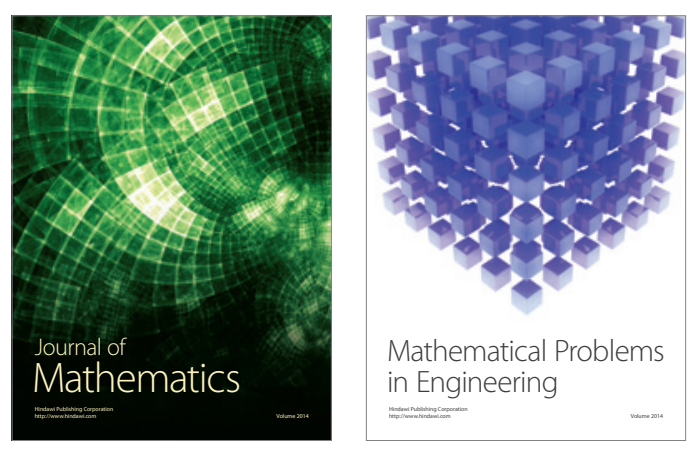

Mathematical Problems in Engineering
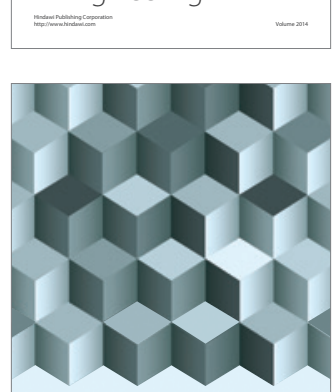

Journal of

Function Spaces
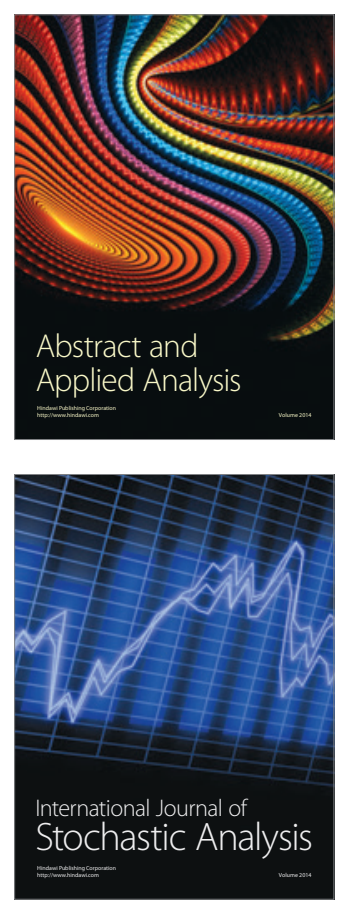

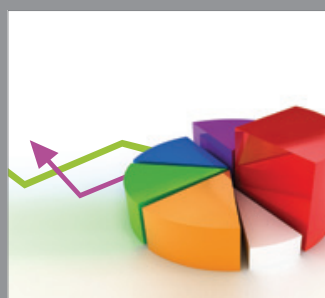

ournal of

Probability and Statistics

Promensencen
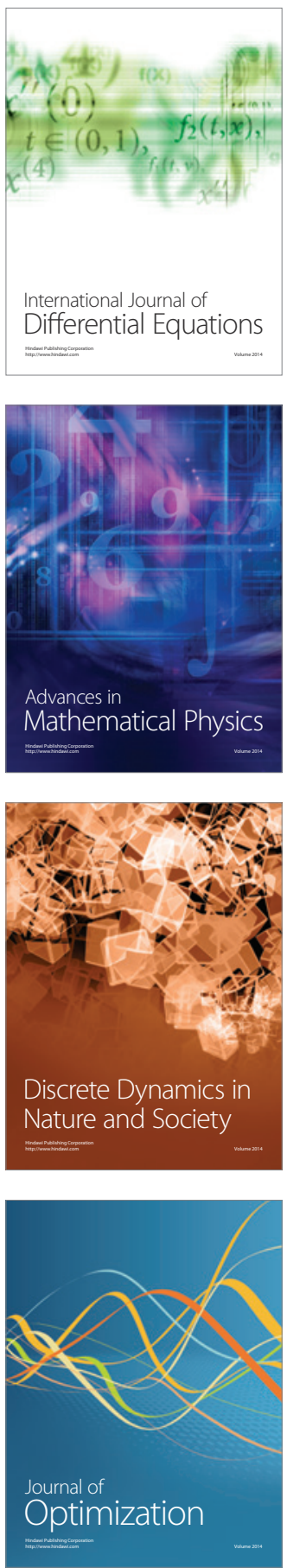